Rev. salud pública. 17 (6): 925-937, 2015

DOI: http://dx.doi.org/10.15446/rsap.v17n6.38368

\title{
Factores relacionados con las prácticas alimentarias de estudiantes de tres universidades de Bogotá
}

\author{
Factors related to students' eating practices in three \\ universities in Bogotá, Colombia
}

\author{
Clara Y. Duarte-Cuervo', Diana M. Ramos-Caballero², \\ Ángela C. Latorre-Guapo ${ }^{3}$ y Pedro N. González-Robayo ${ }^{4}$ \\ 1 Universidad Metropolitana, Barranquilla. Colombia. claradc2002@gmail.com \\ 2 Universidad del Rosario. Bogotá. Colombia. diana.ramos@urosario.edu.co \\ 3 Universidad Nacional de Colombia, Bogotá. Colombia. aclatorreg@unal.edu.co. \\ 4 Universidad Libre. Bogotá, Colombia. pedronelgonzalezrobayo@gmail.com \\ Recibido 8 Junio 2013/Enviado para Modificación 23 Julio 2014/Aceptado 19 Junio 2015
}

\section{RESUMEN}

Objetivo Identificar y describir los hábitos alimentarios de los estudiantes de tres universidades de Bogotá y los factores que influyen en su adopción.

Métodos Se realizaron tres grupos focales con participación promedio de 12 estudiantes de diferentes carreras de pregrado con edades entre 18 y 25 años. El contenido de los relatos fue analizado y categorizado de acuerdo a los factores que influyen en la selección de alimentos y los hábitos de alimentación.

Resultados Los estudiantes señalan los factores sensoriales y de higiene, el costo de los alimentos, la disponibilidad de los mismos y el tiempo disponible para comer como principales factores que influyen en la selección. Se identifica además la influencia de los amigos y la familia, entre otros, en las elecciones en este aspecto. Los estudiantes participantes refirieron preferir la comida de su casa, consumir la mayor parte de sus comidas en el hogar y tener horarios de alimentación marcados por las actividades académicas.

Conclusión Aunque los estudiantes tienen conocimientos acerca de lo que es bueno para ellos en términos de alimentación, en la vida universitaria la organización de las jornadas académicas, la disponibilidad de alimentos y de recursos económicos marcan de manera importante los hábitos y elecciones que realizan. Las estrategias de promoción de hábitos saludables en las universidades deberían entonces privilegiar el control de tales barreras.

Palabras Clave: Preferencias alimentarias, conducta alimentaria, estudiantes, salud escolar (fuente: DeCS, BIREME). 


\section{ABSTRACT}

Objective To identify and describe eating habits of students from three universities in Bogota and factors influencing their adoption.

Methods Three focus groups were formed with an average attendance of 12 students from different undergraduate programs, between the ages of 18 and 25 . The content of the accounts was analyzed and categorized according to the factors influencing the selection of food and eating habits.

Results Students identified sensory and hygiene factors, the cost of food, the availability of food and available time to eat as the main factors influencing food selection. We also identified the influence of friends and family, among others, in their choices. Students who participated reported to prefer food from home, eating most of their meals at home and having eating schedules influenced by academic activities. Conclusion Although students have knowledge about what is good for them in terms of food, in university life, the organization of academic schedule, food availability and economic resources showsignificant influence on their habits and choices. Strategies to promote healthy habits in universities should then prioritize the control of such barriers.

Key Words: Food preferences, eating behavior, Students, Student health services (fuente: MeSH, NLM).

L

os hábitos alimentarios de los estudiantes han despertado especial interés en las instituciones de educación superior en Colombia y otros países, un hallazgo recurrente en los estudios en este campo es que estos se modifican drásticamente con el paso del colegio a la universidad; entre los factores relacionados destacan el tiempo y los recursos económicos $(1,2)$.

Respondiendo a este interés, Nestlé de Colombia y la Universidad del Rosario lanzan en 2008 el concurso de investigación Nutrición, salud y bienestar, dirigido a "apoyar e incentivar la generación de conocimiento alrededor de la salud y el bienestar de las comunidades universitarias" (3), cuyo proyecto ganador fue Caracterización de estilos de vida saludables en estudiantes universitarios, presentado por la Universidad Nacional de Colombia, la Universidad Libre y la Universidad del Rosario.

Durante la primera fase del proyecto se identifica la necesidad de desarrollar estrategias metodológicas que permitan a las instituciones comprender las circunstancias y el contexto en que los estudiantes toman decisiones respecto a su alimentación y así, tener mayores elementos para orientar las acciones de los programas de bienestar. En consecuencia, parte del estudio se orienta a identificar y describir los hábitos alimentarios de 
los estudiantes de las tres universidades y los factores que influyen en su adopción, a partir del análisis de relatos y reflexiones que emergen al usar grupos focales como herramienta metodológica.

Se toma como marco de referencia, desde la antropología nutricional, la propuesta de Pelto, Pelto y Messer (4), quienes plantean que en la selección de los alimentos entran en juego la interrelación entre factores biológicos y culturales. La posición de los autores se sustenta en la teoría que relaciona la nutrición con los procesos socio-culturales, económicos y ecológicos, fundamentada en estudios desde diferentes enfoques antropológicos y con aportes desde la nutrición y las ciencias sociales. Así, “...lo cultural que incluye factores simbólicos, sociales y económicos, en interacción con la información y las preferencias sensoriales, moldean los patrones alimentarios e influencian la selección de alimentos" (4). A partir de estas relaciones, se exponen entonces cinco factores: características sensoriales, económicas y ecológicas; percepción de efectos fisiológicos; dimensión cultural simbólica; y construcción sociocultural de las dietas.

Dentro de las características sensoriales, lo visual, que incluye el tamaño, forma y color, generan juicios de atracción o rechazo; en grupos específicos el color de los alimentos determina si estos son apropiados o no para su consumo. Aunque estas connotaciones se relacionan con la condición de pureza o refinamiento, no necesariamente lo están con lo saludable, lo que es agradable a la vista no siempre es nutritivo, pero puede corresponder a códigos culturales de alimentos adecuados.

Los factores ecológicos como las temporadas de cosecha, las estaciones y los cambios climáticos, también determinan la adquisición de alimentos. Así mismo, los horarios del trabajo y la escuela afectan la elección y el consumo de las comidas durante el día; de la misma manera, la cantidad de tiempo disponible del proveedor o preparador en un hogar, determinan lo que se compra y se prepara para comer. De otra parte, aunque la gente realiza la selección por gusto y factores de conveniencia, el dinero también resulta importante.

En la determinación de los gustos y preferencias relacionados con alimentación, resultan fundamentales las percepciones y reacciones fisiológicas frente a los alimentos, a partir de las cuales las personas los clasifican como buenos o malos, deseables o no, saludables o perjudiciales para su salud. Un claro ejemplo de ello es la saciedad que producen, parece 
haber una relación entre la composición de los alimentos y la saciedad; así, algunas culturas prefieren las proteínas a los vegetales, pues las primeras contribuyen de manera significativa a aumentar esta sensación, mientras que al consumir vegetales, aún en grandes cantidades, la sensación de hambre persiste.

Las personas también suelen clasificar los alimentos de acuerdo a categorías simbólicas construidas culturalmente a partir de información sensorial (subjetiva) y de otro tipo. De acuerdo con los autores, el significado e importancia dado a tales clasificaciones varía según el contexto cultural, pero también de acuerdo a la tendencia de los sujetos a seguir o no las reglas. Así, si bien las estructuras generales de clasificación pueden ser compartidas por los miembros de un grupo, no necesariamente son valoradas e implementadas de la misma manera por todos.

De los factores considerados por Pelto, Pelto y Messer en la dimensión cultural-simbólica, que resultan pertinentes para este estudio, se encuentran: factores de salud, factores de edad y género en la selección o restricción de alimentos, las enfermedades, el estatus económico y el carácter ritual, la estética e imagen corporal.

En relación a la construcción sociocultural de las dietas, se establecen los alimentos apropiados para diferentes momentos del día, el número de comidas y los ingredientes. El consumo de alimentos varía igualmente dependiendo de la temporada del año y las festividades.

Para los autores la selección y consumo de alimentos está determinado, además de los factores individuales, por patrones como la cantidad de veces que se consumen alimentos en el día, los días de ayuno, las fiestas anuales, que en conjunto forman los hábitos alimenticios. La frecuencia y horarios de consumo, los tipos de comida que se ingieren habitualmente o en determinadas ocasiones, las personas con que se comparte en estos momentos, la información transmitida por los medios de comunicación, forman el "código alimentario", que se refiere a la forma para identificar la identidad y los grupos sociales a los que se pertenece, y proporcionan una clave para los criterios de selección individual de los alimentos.

Un estudio sobre patrón alimentario de estudiantes universitarios en México y España (5) describe los hábitos alimentarios en las siguientes categorías: composición de las comidas, frecuencia, lugar, compañía y 
tiempo invertido. Para efectos de este estudio los hábitos alimentarios se describen teniendo en cuenta qué comen los estudiantes-composición de las comidas, con quién lo hacen-compañía, cuántas veces y en qué horarios lo hacen-frecuencia y tiempo invertido, así como dónde lo hacen, por considerarlo una condición que se modifica de manera importante al ingresar a la vida universitaria.

\section{MÉTODOS}

Participaron estudiantes entre 18 y 25 años, matriculados en programas de pregrado de las universidades Libre, del Rosario y Nacional de Bogotá, durante los años 2009 y 2010.

Se utilizó la técnica de grupos focales, teniendo en cuenta que permite procesos de interacción y discusión, generando reacciones que enriquecen la información recolectada, lo que no se presenta con técnicas individuales (6).

En cada universidad se realizaron tres grupos focales de una a dos horas de duración, direccionados por un facilitador, un relator y tres observadores. Se llevaron a cabo en el espacio de asignaturas electivas ofrecidas a diferentes programas académicos de las universidades, buscando la participación de estudiantes de diferentes carreras. El promedio de participantes por grupo fue 12, incluyendo hombres y mujeres (a uno de los grupos sólo asistieron mujeres). Para orientar la construcción de las preguntas que guían los grupos, se hicieron previamente entrevistas exploratorias con estudiantes.

Las discusiones se dieron alrededor de los factores que influyen en la selección de alimentos y los hábitos de consumo. Los relatos se grabaron y transcriben para luego analizar su contenido buscando los elementos redundantes en cada universidad y entre universidades para cada una de las categorías teóricas definidas, se identifican además categorías emergentes.

Los participantes conocen los objetivos y características de la investigación, y deciden participar de manera voluntaria, sin recibir remuneración alguna; se garantiza su anonimato durante todo el proceso.

\section{RESULTADOS}

Los hallazgos se presentan a continuación en términos de factores que influyen en la selección de alimentos y hábitos de alimentación. En lo 
referente al primer tema, se sigue la estructura de categorías propuesta por los autores de referencia, enriquecida con subcategorías emergentes a partir de lo expresado por los estudiantes (Cuadro1).

Factores que influyen en la selección de alimentos

La elección de alimentos en los participantes está condicionada principalmente por factores sensoriales y de higiene. Las mujeres especialmente le dan importancia a la presentación del alimento: "...a mí me gusta ver la comida con mucho color, no me gusta ver un plato simple, todo blanco, amarillo, pálido"; en cuanto a la higiene, refieren: “..,el almuerzo puede tener un aspecto agradable, pero a ti no te consta que fue limpia la preparación...eso produce desconfianza".

De igual forma, otros aspectos tenidos en cuenta son el olor y el sabor, aunque no son tan relevantes para la mayoría de estudiantes en el momento de la elección.

Cuadro 1. Factores que Influyen en la Selección de Alimentos Categorías Iniciales y Emergentes

\begin{tabular}{|c|c|}
\hline Categorías Iniciales & Categorías Emergentes \\
\hline \multicolumn{2}{|l|}{ Características sensoriales } \\
\hline Factores económicos y ecológicos & Origen del alimento \\
\hline Percepción de efectos fisiológicos & $\begin{array}{l}\text { Buenos o malos de acuerdo al tamaño de } \\
\text { las porciones }\end{array}$ \\
\hline Factores cultural simbólicos & $\begin{array}{l}\text { La escuela } \\
\text { La familia } \\
\text { Buenos o malos de acuerdo a la } \\
\text { composición de los alimentos } \\
\text { Higiene }\end{array}$ \\
\hline Construcción sociocultural de las dietas & Creencias \\
\hline
\end{tabular}

Adaptado a partir de las categorías propuestas por Pelto G, Pelto P y Messer E (4).

También se resaltó como un factor influyente el costo de los alimentos, por lo que muchos optan por traerlos de su casa: “...unos traen almuerzo, otros compran, otros no comen nada, depende del horario, de la capacidad económica que uno tenga y de los gustos". En ocasiones se consume lo que las posibilidades económicas permiten, aun cuando se preferiría otro lugar o tipo de alimentación: "cuando estoy ahorrando me como un sandwich con gaseosa, no almuerzo muy bien, pero cuando puedo gastarme la plata pues siempre busco comer algo bueno que tenga carne, ensalada, arroz".

La disponibilidad de alimentos en las universidades y sus alrededores es otro factor que cobra importancia: "...aquí en la universidad uno va a comer y siempre hay pizzas y hamburguesas, lo más saludable que hay es pechuga con ensalada, entonces de lo que hay uno medio come cuando puede y el fin de semana es cuando 
uno se alimenta bien". En este sentido, se evidenció que los estudiantes dan importancia al lugar de donde provienen los alimentos: "no como ensalada en los restaurantes...no les tengo mucha confianza".

Para algunos el consumo de alimentos depende de que un tercero los prepare, incluso que los sirva o los empaque: “....mi hermana me hace el desayuno, desayuno súper bien"; si el proveedor no dispone de tiempo, se busca el alimento fuera: "como en mi casa no me hacían comida, entonces yo salía a algún lado y me comía un perro, una hamburguesa, un sándwich de lo que fuera".

Los estudiantes catalogan los alimentos como buenos o malos, entre otros factores, dependiendo de las sensaciones fisiológicas que les producen y el tamaño de las porciones: “...si a mí me hace daño la leche, yo puedo decir que la leche es mala, pero si yo siento que me da energía, pues yo puedo decir que el alimento es bueno": "...la comida en la universidad es pésima porque uno paga muchísimo y la porción que sirven es deplorable. Alrededor es mejor, las porciones son más grandes... dan mejor cantidad de proteína, vegetales, carbohidratos". Los hombres además le dan especial importancia a la sensación de saciedad: "uno piensa en llenarse y no en lo que está comiendo, es la forma que uno tiene de ver la comida, cuando uno dice tengo hambre y le dicen hay fruta, uno no la consume porque sabe que eso no lo va a llenar". Estas sensaciones también determinan los horarios que se consideran adecuados para comer ciertos tipos de alimentos: "si como mucho por la noche, al otro día amanezco mal o por la noche no puedo dormir bien".

Otro factor que influye para determinar si los alimentos son buenos o malos son los conocimientos construidos alrededor de su composición nutricional: "...hay unos alimentos que funcionan como energéticos, que son como la gasolina del carro y otros que son como la estructura del carro. Dentro de la estructura están las grasas, proteínas y carbohidratos...", muchos de los cuales han sido adquiridos en la escuela y a través de la familia: "es la costumbre y la educación que a uno le van dando desde pequeño, le van diciendo que las comidas malas son las hamburguesas o las comidas rápidas, mientras las comidas buenas son cosas como las verduras, pero uno no lo sabe porque lo estudió a fondo sino porque cuando uno va creciendo le van diciendo esto es bueno, esto es malo, esto es lo que se debe comer y esto no".

Identifican como buenos los alimentos de origen natural, las frutas y las verduras, también reconocen la forma de preparación, el balance y la cantidad en el consumo como características con efectos positivos para su salud, y en general, se asocian las comidas rápidas y las grasas con efectos negativos. Refieren que el exceso en el consumo de ciertos alimentos 
puede generar enfermedades, algunas de sus prácticas alimentarias están mediadas por el hecho de padecer una enfermedad: "el exceso de ciertos alimentos es malo, muchos dulces puede provocar una diabetes": "sufro del colon y de gastritis, tengo que comer bien...".

La construcción de las dietas se ve influenciada por los medios de comunicación, principalmente la televisión. Frente a esto resaltan tres opiniones, primero reconocen que las mujeres incluyen o excluyen alimentos dependiendo de lo que recomienden o rechacen personajes públicos; segundo, manifiestan que los mensajes transmitidos por la televisión generan que las personas clasifiquen las comidas como buenas y malas; por último, mencionan que por éste medio se transmiten mensajes contradictorios: "por una parte se proyectan comerciales que invitan al consumo de comidas rápidas ricas en grasa y por otra se ofertan productos para bajar de peso".

Los estudiantes reconocen además la influencia de otras personas en sus preferencias alimentarias en la universidad. Por una parte, de sus amigos y compañeros: "ella come a cada rato y yo también; traigo almuerzo porque ella también trae"; de otro lado la pertenencia a grupos culturales o étnicos también interviene, así, las costumbres del país o la región y más aún de la familia, son determinantes: "Yo soy de Cali y el tinto en la mañana es sagrado"; "en la casa todos somos adictos al pan, no puede faltar el pan al desayuno".

La construcción de la dieta también responde al tiempo disponible para comer: "al almuerzo por lo general tengo una sola hora, entonces como lo que encuentro que por lo general es comida rápida"; así como a algunas creencias que se van adoptando con el tiempo: "hay una serie de mitos interesantes, como que al consumir carbohidratos no se va a desarrollar la masa muscular".

Hábitos de alimentación

Los resultados se presentan en términos de los alimentos que consumen, cuántas veces al día y en qué horarios, dónde lo hacen y con quién.

Al indagar sobre los alimentos que consumen habitualmente, se encontró que al desayuno suelen incluir una proteína, por lo general huevo, un carbohidrato como pan, arepas o galletas, una bebida láctea generalmente caliente, chocolate o café, o fría como yogurt o leche. Otros estudiantes comen frutas, queso y cereal, en casos particulares sándwich, caldo o salchicha. Varias personas refieren no desayunar. El almuerzo suele estar compuesto por un cereal, generalmente arroz, proteína 
animal como carne o pollo y vegetal como fríjol, garbanzo o lenteja y tubérculos como papa o yuca. Respecto al consumo de verduras, hacen referencia a la ensalada como parte del almuerzo, pero es un alimento que prefieren consumir en casa. Otros almuerzan comidas rápidas como pizza, hamburguesa y empanadas. En la cena se distinguen tres grupos, unos que consumen alimentos y cantidades similares a los del almuerzo, otros que prefieren alimentos que consideran "más ligeros" como café con pan, pasta o un sándwich, y otros que mencionan no consumir nada. Entre tanto para los refrigerios, prefieren gaseosas, paquetes de papas, chocolates, pasteles, empanadas o en algunos casos frutas; los fines de semana los hábitos de consumo varían en relación a tipos de alimentos y horarios.

Algunos estudiantes acostumbran comer cinco veces al día, tres comidas principales y dos refrigerios, otros solo desayuno, almuerzo y comida. El número de comidas y los horarios son regulados por las actividades académicas, estas se modifican en la transición del colegio a la universidad volviéndose irregulares, por lo cual refieren no tener o tener muy pocos horarios fijos para alimentarse: "...antes comía 5 veces al día pero por tiempo me tocó modificarlo"; particularmente la hora de almuerzo suele afectarse y con frecuencia se posterga hasta el momento de llegar a la casa: "...tipo 6 de la tarde me como lo que hayan hecho de almuerzo en mi casa"; como consecuencia, se refiere comer en mayor cantidad en las noches: "...el único horario fijo que tengo es la comida, de resto me la paso comiendo todo el día, y es por ahí a las 8". Se evidencia que el espacio doméstico es el preferente para consumir los alimentos, pero en ocasiones las distancias influyen en los horarios de ingesta. En algunos casos los horarios se regulan por prescripción profesional: “...por mi hipoglicemia intento comer 5 veces al día".

El lugar donde los estudiantes toman sus alimentos está influenciado por las actividades académicas y en ocasiones laborales. El desayuno y la cena son consumidos en casa, el primero en ocasiones se hace en la universidad: "Yo si no desayuno en la casa porque salgo a las 5 de la mañana, entonces cuando llego a la universidad ahí sí como algo". El almuerzo suele ser en la universidad o sus alrededores. En general refieren consumir alimentos en compañía de sus amigos, quienes a su vez influyen en hábitos como si se come o no, el tipo y calidad de alimentos que se consumen y el lugar: “....yo comía súper mal... pero ahora que me junto con ella, ella come a cada rato y yo también, traigo almuerzo porque ella también trae, depende de con quién estés". 


\section{DISCUSIÓN}

Dentro de los factores que influyen en la selección de alimentos de los universitarios, en este estudio se destacan el tiempo disponible, los recursos económicos, la influencia de otros y la presentación de los alimentos. Los dos primeros se destacan en la literatura entre los aspectos más relevantes $(1,7,8)$, el tiempo disponible de acuerdo a las cargas académicas determina el tipo y la cantidad de alimentos que se consumen, a mayor disponibilidad de tiempo eligen comidas más abundantes y nutritivas y prefieren tomarlas en casa, en caso contrario se prefieren las comidas rápidas y los paquetes. La selección de estos alimentos también obedece a factores económicos, los estudiantes asocian los alimentos buenos y nutritivos con precios fuera de su alcance; solo en algunos casos la falta de tiempo o dinero los lleva a no comer.

El entorno social también se evidenció como un factor que influye de manera importante en la selección de alimentos por parte de los estudiantes, en este sentido se resaltaron los amigos, la familia, los medios de comunicación y las indicaciones dadas por profesionales de la salud, lo que coincide con otros estudios similares (1), aunque en otras investigaciones con poblaciones similares, no se dio tanta importancia a este factor (8). Los compañeros determinan con frecuencia no sólo qué se consume sino dónde hacerlo (9); hábitos como traer comida de la casa, o comprarla y consumir ciertos alimentos se comparten y se consolidan en los grupos. Entre tanto, los saberes aprendidos en la familia y de los profesionales respecto a la alimentación, se reproducen y se transforman en la vida cotidiana de los estudiantes, generando cambios en las rutinas que en la infancia y la adolescencia temprana se mantienen gracias a los horarios poco variables de la escuela.

Finalmente, la percepción visual resulta también determinante para los universitarios, cobran importancia la presentación de los alimentos, la higiene de los establecimientos y del personal que los atiende (10), siendo esto más evidente en los relatos de las mujeres, mientras que en los de los hombres sobresalen las referencias a la cantidad. Así, dichas percepciones se asocian para las mujeres con la composición nutricional de los alimentos $(11,12)$ y para los hombres, con la posibilidad de generar sensaciones de saciedad. En contraposición a otros estudios, el sabor no resultó ser uno de los factores más destacados a la hora de seleccionar los alimentos (8). 
En relación con los hábitos alimenticios de los estudiantes se resaltan dos aspectos, dónde consumen sus alimentos y cuándo lo hacen. Respecto al lugar se destaca la preferencia por la comida de la casa, lo que determina la hora de consumo, esto es más frecuente entre quienes viven cerca a sus lugares de estudio y cuentan con un proveedor en casa, en este sentido, otros estudios en universitarios han encontrado la preferencia de los estudiantes por consumir el desayuno y la cena en el hogar $(1,13)$. No obstante, dadas las largas jornadas de estudio y la distancia a las viviendas, se hace necesario el consumo de alimentos en la universidad, especialmente el almuerzo, aun cuando los estudiantes manifiestan inconformidad con la oferta, la calidad y los precios del entorno universitario (1).

Otros autores también resaltan el hábito de los universitarios de omitir comidas, especialmente el desayuno $(1,8,13)$. Al respecto, resulta importante destacar los hallazgos del estudio de la Universidad del Bosque (1), en el que se encontró que es frecuente el hábito de no desayunar en los jóvenes y que éste se incrementa levemente con el ingreso a la universidad. No obstante, en este estudio los estudiantes reconocen la importancia de esta comida, considerándola incluso indispensable para su funcionamiento cotidiano, esto muestra que son conscientes y están informados sobre los beneficios de tener buenos hábitos alimentarios, pero encuentran barreras para ponerlos en práctica.

Todo lo anterior lleva a identificar la utilidad de este tipo de estudios para ampliar y complementar la información que se obtiene tradicionalmente a través de estudios cuantitativos, que utilizan instrumentos como encuestas o diarios de consumo de alimentos; en la medida que permiten rescatar datos que generalmente no se valoran, dando así la posibilidad de aproximarse más a la cotidianidad de los universitarios y brindando a los gestores de programas, mayores herramientas para cualificar sus intervenciones.

En este sentido sería útil avanzar en investigaciones dirigidas a la identificación de barreras para la implementación de estilos de vida saludables en los universitarios las cuales, si bien surgieron en el análisis durante este estudio, no se exploraron a profundidad. Adicionalmente, teniendo en cuenta que durante este estudio se evidenció que en el grupo focal conformado sólo por mujeres, las discusiones y las narraciones de experiencias personales relacionadas con la alimentación se generaron con mayor facilidad, se recomienda para investigaciones similares conformar grupos por género. 
Para terminar, los hallazgos de este estudio, así como de otros similares $(11,14)$ han evidenciado que los estudiantes universitarios tienen conocimiento y valoran la alimentación saludable; por lo tanto, los programas dirigidos a promocionar hábitos alimentarios adecuados, más allá de privilegiar estrategias educativas, deberían dirigirse de manera más contundente a controlar barreras como la oferta, los precios, la manipulación e higiene y la presentación de los alimentos en los establecimientos dentro y en los alrededores de los centros educativos, así como la escasa disponibilidad de tiempo para la alimentación, generada por las formas de organización de la carga académica •

Agradecimientos: A Nestlé de Colombia, financiadora de este estudio; a los grupos de investigación Centro de Estudios para la Medición de la Actividad Física CEMA de la Universidad del Rosario, y Actividad Física y Salud de la Universidad Libre; al Programa de Promoción de Actividad Física Vitalízate de la Universidad Nacional de Colombia; al psicólogo Fernando Rivera, por su participación durante la investigación, y a los estudiantes de las tres universidades.

Conflicto de intereses: Los autores manifiestan que son independientes con respecto a la institución financiadora y que durante la ejecución del trabajo y la redacción del manuscrito no han incidido intereses o valores distintos a los de la investigación.

\section{REFERENCIAS}

1. Saad C, Ibañez E, León C, Colmenares C, Vega N, Díaz Y. Cambios en los hábitos alimentarios de los estudiantes de enfermería de la Universidad El Bosque durante el proceso de formación académica, Bogotá. D.C. 2007. Rev Col Enfermería. 2008;3(3):51-60.

2. Ibañez E, Thomas Y, Bicenty A, Barrera J, Martínez J, Gerena R. Cambios de hábitos alimentarios de los estudiantes de odontología de la Fundación Universitaria San Martín de Bogotá, Colombia. Nova - publicación científica en Ciencias Biomédicas. 2008 Junio;6(9):101-112

3. Ministerio de Educación Nacional. [Internet]. Disponible en: http://www.mineducacion. gov.co/cvn/1665/w3-article-207844.html. Consultado mayo de 2013.

4. Messer E, Pelto G, Pelto P. Research Methods in Nutritional Anthropology. Tokio, Japón: The United Nations University Press; 1989.

5. Díaz M, Riba M, Rodríguez AM, Mora MT. Patrón alimentario de estudiantes universitarios: Comparación entre culturas. Rev Esp Nutrición Comunitaria. 2005; 1(11):8-11

6. Onwuegbuzie AJ, Dickinson WB, Leech L, Zoran AG. Un marco cualitativo para la recolección y análisis de datos en la investigación basada en grupos focales. Paradigmas. 2011; 3(2):127-157.

7. Larson NI, Perry CL, Story M, et al. Food preparation by young adults is associated with better diet quality. J Am Diet Assoc. 2006;106:2001-2007. 
8. Driskell JA, Kim YN, Goebel KJ. Few Differences Found in the Typical Eating and Physical Activity Habits of Lower-Level and Upper-Level University Students. J Am Diet Assoc. 2005; 105(5): 798-801.

9. Tobar F, Vásquez S, Bautista LF. Descripción de hábitos y comportamientos alimentarios de los estudiantes de la Facultad de Ciencias de la Pontificia Universidad Javeriana. Universitas Scientiarum. 2005; 13(1):55-63.

10. Jiménez M, Álvarez G, Jiménez M. Patrón Alimentario y criterios individuales que influencian en la selección de alimentos en estudiantes de ciclos básicos de la licenciatura de medicina de la FES IZTACALA. En: Libro de resúmenes del II Congreso Internacional de Educación Médica. Benito Juárez. Distrito Federal, México; 2010. p. 231.

11. Morse KL, Driskell JA. Observed sex differences in fast-food consumption and nutrition self-assessments and beliefs of college students. Nutr Res. 2009; 29:173-179

12. Temperio A, Burns C, Cameron SD, Crawford D. Fattening foods perceptions and misconceptions: aqualitative and quantitative. Nutr Diet. 2003;60(4):230-238.

13. Vargas $M$, Becerra F, Prietro E. Evaluación de la ingesta dietética en estudiantes universitarios. Bogotá, Colombia. Rev. Salud Pública (Bogotá). 2010; 12(1):116-125

14. Montero A, Úbeda N, García A. Evaluación de hábitos alimentarios de una población de estudiantes universitarios en relación con sus conocimientos nutricionales. Nutr Hosp. 2006; 21(4):466-473 